\title{
On the Positive Bias of Peak Horizontal Velocity from an Idealized Doppler Profiler
}

\author{
David A. Short ${ }^{1}$ and Francis J. Merceret ${ }^{2}$ \\ ${ }^{1}$ ENSCO, Inc., Cocoa Beach, Florida \\ ${ }^{2}$ Applied Meteorology Unit, NASA / Kennedy Space Center, Florida
}

Submitted to Journal of Atmospheric and Oceanic Technology

. January 2004

Corresponding Author Address: David A. Short, ENSCO, Inc., 1980 N. Atlantic Ave., Suite 230, Cocoa Beach, Florida, 32931. Email: short.david@ensco.com. 


\begin{abstract}
In the presence of 3-D turbulence, peak horizontal velocity estimates from an idealized Doppler profiler are found to be positively biased due to an incomplete specification of the vertical velocity field. The magnitude of the bias was estimated by assuming that the vertical and horizontal velocities can be separated into average and perturbation values and that the vertical and horizontal velocity perturbations are normally distributed. Under these assumptions, properties of the Type-I Extreme Value Distribution for maxima, known as the Gumbel distribution, can be used to obtain an analytical solution of the bias. The bias depends on geometric properties of the profiler configuration, the variance in the horizontal velocity, and the unresolved variance in the vertical velocity. When these variances are normalized by the average horizontal velocity, the bias can be mapped as a simple function of the normalized variances.
\end{abstract}




\section{Introduction}

Doppler profilers are used to obtain estimates of horizontal (U) and vertical velocity $(W)$ within the atmosphere (Van Zandt 2000) and under water (Woodward and Appell 1986) using acoustic, radar and optical remote sensing techniques. Electromagnetic or acoustic energy at a known frequency is transmitted into the medium of interest and the frequency of the back-scattered energy is measured by a directional receiver. Receiver characteristics such as size and shape define its beam, typically a narrow cone projecting away from the receiver throughout some depth of the fluid. The difference between the transmitted and received frequencies, referred to as the Doppler shift, is used to estimate the velocity component of back-scatterers along the beam axis. The back-scattering elements are assumed to be passive tracers of the fluid motion and the estimated velocity component along the beam axis is referred to as the radial velocity $(R)$. Sophisticated transmitter/receiver configurations and signal generating/processing techniques have been developed over the past several decades to maximize the accuracy of $R$ estimates and subsequent retrieved $U$ and $W$.

The average retrieved $U\left(\overline{U_{\mathrm{Ret}}}\right)$ from Doppler profiling systems has been validated extensively with in situ observing systems in both the atmosphere (May et al. 1989 ; Crescenti 1997) and underwater (Chereskin et al. 1987; Gilboy et al. 2000). $\overline{U_{\mathrm{Ret}}}$ is generally well-estimated for averaging times of 15 minutes or greater. Average measures of turbulence can also be derived from Doppler profiler observations by statistical/dynamical methods (Kramar and Kouznetsov 2002; Lu and Lueck 1999). However, the precision of the retrieved instantaneous $U\left(\left(\bar{U}+u^{\prime}\right)_{\mathrm{Re}}\right)$, where $u^{\prime}$ implies 
a perturbation from $\bar{U}$ with a time scale of a few seconds, is more problematic. As a result, the accuracy of the retrieved peak $U\left(\left(U_{n}^{\oplus}\right)_{\text {Ret }}\right)$ from a collection of $\left(\bar{U}+u^{\prime}\right)_{\text {Ret }}$ of size $\mathrm{n}$, can be significantly affected.

This paper describes an idealized Doppler profiler in an idealized fluid, where the true instantaneous $R\left(\left(\bar{R}+r^{\prime}\right)_{\text {True }}\right)$ is composed of weighted sums of $\left(\bar{U}+u^{\prime}\right)_{\text {True }}$ and $\left(\bar{W}+w^{\prime}\right)_{\text {True }}$, with weights dependent on the beam configuration. In the case of 3-D turbulent flow the typical beam configuration does not provide adequate information for an accurate estimate of $\left(\bar{U}+u^{\prime}\right)_{\text {Ret }}$, although $\overline{\left(\bar{U}+u^{\prime}\right)_{\text {Ret }}}$ can be shown to be unbiased under certain assumptions. For applications where $U_{n}^{\oplus}$ from a collection of $\bar{U}+u^{\prime}$ of size $\mathrm{n}$ is of interest, errors in $\left(\bar{U}+u^{\prime}\right)_{\text {Ret }}$ propagate into $\left(U_{n}^{\oplus}\right)_{\operatorname{Ret}}$ and, in general, introduce a positive bias in the average peak value $\overline{\left(U_{n}^{\oplus}\right)_{\text {Ret }}}$. Section 2 describes an idealized profiler configuration along with retrieval algorithms for uniform and turbulent flows. Section 3 uses Extreme Value Theory to provide analytical solutions to $\sqrt[\left(U_{n}^{\oplus}\right)_{\text {Ret }}]{\text { and }}$ $\widehat{\left(U_{n}^{\oplus}\right)_{\text {True }}}$ as a function of turbulent properties of the fluid and the profiler configuration. Section 4 provides a summary and conclusions. A list of symbols is given in appendix A.

\section{An Idealized Doppler Profiler}

The following description of an idealized Doppler profiler is intended to represent, in the simplest terms, how $\left(\bar{U}+u^{\prime}\right)_{\text {True }}$ and $\left(\bar{W}+w^{\prime}\right)_{\text {True }}$ combine to form $\left(\bar{R}+r^{\prime}\right)_{\text {True }}$ from the oblique and vertical beams of a typical system. The $\left(\bar{R}+r^{\prime}\right)_{\text {True }}$ are then used to obtain $\left(\bar{U}+u^{\prime}\right)_{\text {Ret }}$. For conditions where the $\left(\bar{W}+w^{\prime}\right)_{\text {True }}$ varies in space and/or time, 
$\left(\bar{U}+u^{\prime}\right)_{\operatorname{Ret}}$ is shown to be susceptible to an error that is directly proportional to the difference in $\left(\bar{W}+w^{\prime}\right)_{\text {True }}$ over the oblique and vertical beams. A statistical modeling approach is used to show how this difference affects $\overline{\left(U_{n}^{\oplus}\right)_{\text {Ret }}}$. The statistical model quantifies three important characteristics: 1) $\overline{\left(\bar{U}+u^{\prime}\right)_{\text {Ret }}}$ is unbiased under reasonable assumptions, 2) $\overline{\left(U_{n}^{\oplus}\right)_{R e t}}$ is positively biased under the same assumptions, and 3) The magnitude of the positive bias is dependent on the unresolved temporal and/or spatial variations of $\left(\bar{W}+w^{\prime}\right)_{\text {True }}$, expressed in $w_{2}^{\prime}-w_{1}^{\prime}$.

Consider an idealized Doppler profiler that measures the $\left(\bar{R}+r^{\prime}\right)_{T r u e}$ along each of two beams, $b_{1}$ and $b_{2}$, as in Figure 1 . The $b_{1}$-beam is vertically oriented with respect to the local horizontal plane. The $b_{2}$-beam is oriented at an angle $\Theta$ from the vertical in order to obtain information about $U$. While the typical Doppler profiling system has three or more beams to resolve the three orthogonal velocity components, the essence of the mathematical and statistical arguments supporting a positive bias in $\overline{\left(U_{n}^{\oplus}\right)_{\operatorname{Ret}}}$ can be readily developed for a two-beam system and are generally applicable for a multi-beam system.

The profiler obtains doublets of $\left(\bar{R}+r^{\prime}\right)_{T r u e}, \mathbf{V}_{1}\left(D_{1}\right)$ and $\mathbf{V}_{2}\left(D_{2}\right)$, where the symbols $D_{1}$ and $D_{2}$ denote distances from point $\mathbf{p}$ in Figure 1, the location of the instrument. The condition $D_{1}=D_{2} \cdot \operatorname{Cos}(\Theta)$ is required to obtain radial velocities from both beams at the same height, $H=D_{1}$, above the local horizontal plane. The following notation was developed by assuming this condition was met and dropping the notation for height and distance. Note also that $\Theta$ is typically about $15^{\circ}$ for atmospheric systems and 
$30^{\circ}$ for underwater systems in order to obtain profile information over a useful depth and to avoid $b_{2}$ side-lobe contamination from ground targets with zero-Doppler shift. Because $\Theta$ is small, $\mathbf{V}_{2}$ can be significantly affected by $\left(\bar{W}+w^{\prime}\right)_{\text {True }}$.

\section{a. Equations for a Uniform Velocity Field with no Turbulence}

Under idealized conditions of temporal and spatial uniformity in the velocity field, the idealized profiler observations are described by the following equations (Peterson 1988):

$$
\begin{aligned}
& \mathbf{V}_{1}=(W)_{\text {True }} \\
& \mathbf{V}_{\mathbf{2}}=(U)_{\text {True }} \cdot \sin (\Theta)+(W)_{\text {True }} \cdot \cos (\Theta)
\end{aligned}
$$

Equation 2 can be directly solved for $(U)_{\text {True }}$ as follows:

$$
(U)_{\text {True }}=\mathbf{V}_{2} \cdot \sec (\Theta)-(W)_{\text {True }} \cdot \cot (\Theta)
$$

There are three important points to note from Equations 1-3:

1. Equation 1 shows that $(W)_{\text {True }}$ is obtained from $\mathbf{V}_{\mathbf{1}}$, the radial velocity measured by the vertically oriented $b_{1}$-beam.

2. $(W)_{\text {True }}$ appears in the second term for the $(U)_{\text {True }}$ solution in Equation 3. The second term makes a correction for the effect of $(W)_{\text {True }}$ on $\mathbf{V}_{2}$.

3. The correction term is amplified by the cotangent of the oblique beam angle, $\Theta$. For $\Theta=15^{\circ}, \cot \left(15^{\circ}\right)=3.73$ and $\sec \left(15^{\circ}\right)=3.86$. For $\Theta=30^{\circ}, \cot \left(30^{\circ}\right)$ $=1.73$ and $\sec \left(30^{\circ}\right)=2$. 


\section{b. Equations for a Turbulent Velocity Field}

Consider a turbulent fluid where the $(W)_{\text {True }}$ and $(U)_{\text {True }}$ can be expressed in terms of average $\left({ }^{-}\right)$and perturbation $(')$ values. $\left(\bar{W}+w^{\prime}\right)_{\text {True }}$ over the $b_{1}$ - and $b_{2}$-beams will be denoted by $\mathbf{W}_{1}=\overline{W_{1}}+w_{1}^{\prime}$ and $\mathbf{W}_{2 .}=\overline{W_{2}}+w_{2}^{\prime}$, respectively, where $\overline{W_{1}}=\overline{W_{2}}=0$. The $\left(\dot{\bar{U}}+u^{\prime}\right)_{T r u e}$ over the $b_{2}$-beam will be denoted by $\overline{U_{2}}+u_{2}^{\prime}$, where $\overline{U_{2}}>0$ will be assumed. In order to characterize a retrieval algorithm for these turbulent conditions, consider the following revised formulation of the profiler observations:

$$
\begin{aligned}
& \mathbf{V}_{1}=w_{1}^{\prime}, \\
& \mathbf{V}_{2}=\left(\bar{U}_{2}+u_{2}^{\prime}\right)_{\text {True }} \cdot \sin (\Theta)+w_{2}^{\prime} \cdot \cos (\Theta) .
\end{aligned}
$$

The appropriate solution for the true horizontal velocity would be

$$
\left(\bar{U}_{2}+u_{2}^{\prime}\right)_{\text {True }}=\mathbf{V}_{2} \cdot \sec (\Theta)-w_{2}^{\prime} \cdot \cot (\Theta) .
$$

However, in general, $w_{2}^{\prime}$ is not observed. It is approximated by $w_{1}^{\prime}$, as in the following equation for the retrieved horizontal velocity:

$$
\left(\bar{U}_{2}+u_{2}^{\prime}\right)_{\mathrm{Re} t}=\mathrm{V}_{2} \cdot \sec (\Theta)-w_{1}^{\prime} \cdot \cot (\Theta)
$$

$\left(\bar{U}_{2}+u_{2}^{\prime}\right)_{\mathrm{Ret}}$ can be expressed as the true horizontal velocity, $\left(\bar{U}_{2}+u_{2}^{\prime}\right)_{T r u e}$, plus an error term, by combining Equations 6 and 7 .

$$
\left(\bar{U}_{2}+u_{2}^{\prime}\right)_{\text {Ret }}=\left(\bar{U}_{2}+u_{2}^{\prime}\right)_{\text {True }}+\left(w_{2}^{\prime}-w_{1}^{\prime}\right) \cdot \cot (\Theta)
$$

The error term in Equation 8 is composed of the difference between the perturbation vertical velocities from the two beams, amplified by the cotangent $(\Theta)$ factor. It is useful to note that a similar error term would exist in Equation 8 for a retrieval algorithm that did not correct for $w_{1}^{\prime}$ in Equation 7. Measurement errors in $\mathbf{V}_{\mathbf{1}}$ and $\mathbf{V}_{2}$ would generate 
additional error terms. $\left(\bar{U}_{2}+u_{2}^{\prime}\right)_{\operatorname{Ret}}$ will be affected by the error term because turbulent eddies cause the vertical velocity to vary rapidly in time and space, resulting in $w_{2}^{\prime} \neq w_{1}^{\prime}$. As a result, $\left(\bar{U}_{2}+u_{2}^{\prime}\right)_{\text {Ret }}$. can be expected to be more variable than $\left(\bar{U}_{2}+u_{2}^{\prime}\right)_{\text {True }}$. However, $\overline{\left(\bar{U}_{2}+u_{2}^{\prime}\right)_{\text {Ret }}}$ will be unbiased with respect to $\overline{\left(\bar{U}_{2}+u_{2}^{\prime}\right)_{\text {True }}}$ if $\overline{w_{2}^{\prime}-w_{1}^{\prime}}$ is zero and if variations in the error term are uncorrelated with perturbations in $\left(\bar{U}_{2}\right)_{\text {True }}$ :

On the other hand, $\left(U_{n}^{\oplus}\right)_{\text {Ret }}$ may be systematically biased if positive peaks in the error term coincide with peak or near-peak values in $\left(\bar{U}_{2}+u_{2}^{\prime}\right)_{\text {True }}$. The probability of such coincidence would increase under one or more of the following three conditions: 1) As the averaging interval becomes long, 2) As the time-scale of $w^{\prime}$ variations becomes short compared to the time-interval between observations, or 3) As the distance between the beams becomes large compared to the spatial scale of the turbulent eddies. Some quantitative insights into these potential errors of $\left(U_{n}^{\oplus}\right)_{\text {Ret }}$ can be obtained by use of a statistical model, as described below, to simulate Doppler profiler observations and the resulting $\left(\bar{U}_{2}+u_{2}^{\prime}\right)_{\text {Ret }}$.

\section{Statistical Modeling of Peak Horizontal Velocity Bias}

The idealized profiler concept introduced in Section 2 will be used here to obtain quantitative insights into the statistics of $\left(U_{n}^{\oplus}\right)_{\text {rrue }}$ and $\left(U_{n}^{\oplus}\right)_{\text {Ret }}$ by employing analytical properties of the Extreme Value Type-I distribution for maxima (the Gumbel distribution). 
A Gumbel distribution can be obtained by generating random samples of size $\mathrm{n}$ from a normal distribution, then extracting the maximum value from each sample and repeating the process ad infinitum (Coles 2001). The maxima will have a Gumbel distribution. In the present case, $\left(\overline{U_{2}}+u_{2}^{\prime}\right)_{\text {True }}$ is assumed to be normally distributed about $\overline{U_{2}}$, with a standard deviation of $\sigma$ (Mitsuta and Tsukamoto 1989). In a thought experiment we imagine that from each random sample of size $\mathrm{n}$ from the population of $\left(\overline{U_{2}}+u_{2}^{\prime}\right)_{T \text { Tre }}$, the peak, or maximum, value is selected and used to create a population of $\left(U_{n}^{\oplus}\right)_{\text {True }}$. The distribution of $\left(U_{n}^{\oplus}\right)_{\text {True }}$ will be Gumbel in form with scale and location parameters $\lambda_{\text {True }}$ and $\xi_{\text {True }}$, respectively. At the same time a sample of $\left(\overline{U_{2}}+u_{2}^{\prime}\right)_{\text {Ret }}$ is generated consisting of $\left(\overline{U_{2}}+u_{2}^{\prime}\right)_{\text {True }}$ plus the random error term as described by Equation 8. The error term comes from normal distributions of $w_{2}^{\prime}$ and $w_{1}^{\prime}$ with zero means and equal variances. A corresponding distribution of $\left(U_{n}^{\oplus}\right)_{\text {Ret }}$ is generated by taking the maximum $\left(\overline{U_{2}}+u_{2}^{\prime}\right)_{\text {Ret }}$ for each sample of size $\mathrm{n}$. When the error term is normally distributed and independent of $\left(\overline{U_{2}}+u_{2}^{\prime}\right)_{\text {True }}$, the resulting distribution of $\left(U_{n}^{\oplus}\right)_{\text {Ret }}$ will also be Gumbel in form with parameters $\lambda_{\text {Ret }}$ and $\xi_{\text {Ret }}$. The equations below describe how $\lambda_{\text {True }}, \xi_{\text {True }}, \lambda_{\text {Ret }}$ and $\xi_{\text {Ret }}$ depend on $\mathrm{n}, \overline{U_{2}}, \sigma$, and parameters of the error term. The error term will have a variance that depends on properties of $\left(w_{2}^{\prime}-w_{1}^{\prime}\right)$ and the amplifying $\cot (\Theta)$ factor.

The Gumbel PDF is given by

$$
\mathbf{G}\left(U^{\oplus}\right)=1 / \lambda \cdot \exp \left\{-\left(U^{\oplus}-\xi\right) / \lambda\right\} \cdot \exp \left[-\exp \left\{-\left(U^{\oplus}-\xi\right) / \lambda\right\}\right] \cdot(9)
$$

The average value and variance of the maxima, $U^{\oplus}$ in this case, are given by 


$$
\begin{aligned}
& \left(U^{\oplus}\right)=\xi+\lambda \cdot 0.5772 \text { and } \\
& \operatorname{Var}\left[U^{\oplus}\right]=\lambda^{2} \cdot \pi^{2} / 6,
\end{aligned}
$$

respectively. The value 0.5772 is an approximation of Euler's constant (Abramowitz and Stegun 1965), hereafter Eu.

When $\overline{U_{2}}$ and $\sigma_{\text {True }}$ are the mean and standard deviation of $\left(\bar{U}_{2}+u_{2}\right)_{T r u e}$, the parameters of the resulting Gumbel PDF of $\left(U_{n}^{\oplus}\right)_{\text {True }}$ are

$$
\begin{aligned}
& \xi_{\text {True }}=\overline{U_{2}}+\mathbf{a}_{\mathbf{n}} \cdot \sigma_{\text {True }} \quad \text { and } \\
& \lambda_{\mathrm{T}}=\mathbf{b}_{\mathbf{n}} \cdot \sigma_{\text {True }},
\end{aligned}
$$

where, $\mathbf{a}_{\mathbf{n}}$ and $\mathbf{b}_{\mathrm{n}}$; known as normalizing constants, are dependent on the sample size and are given by (T. Rolf Turner, University of New Brunswick, Canada, personal communication):

$$
\begin{aligned}
& a_{n}=\operatorname{sqrt}[2 \cdot \ln (n)]-[\ln (4 \pi)+\ln (\ln (n))] /[2 \cdot \operatorname{sqrt}(2 \cdot \ln (n))] \\
& b_{n}=1 / \operatorname{sqrt}[2 \cdot \ln (n)] .
\end{aligned}
$$

The average value of $\left(U_{n}^{\oplus}\right)_{\text {True }}$ can then be written in terms of parameters of the underlying normal distribution and the normalizing constants by

$$
\overline{\left(U_{n}^{\oplus}\right)_{\text {True }}}=\overline{U_{2}}+\sigma_{\text {True }} \cdot\left(\mathbf{a}_{\mathbf{n}}+\mathbf{E}_{\mathbf{u}} \mathbf{b}_{\mathbf{n}}\right) .
$$

The $\left(\mathbf{a}_{\mathbf{n}}+\mathbf{E}_{\mathbf{u}} \mathbf{b}_{\mathbf{n}}\right)$ factor, hereafter $\mathbf{G}_{\mathbf{C}}$, is weakly dependent on $\mathrm{n}$, changing from 2.56 to 2.92 as $\mathrm{n}$ goes from 100 to 300 . For example, consider $\overline{U_{2}}=10, \sigma_{\text {True }}=2.0$, and $\mathrm{n}=100$.

We find $\overline{\left(U_{n}^{\oplus}\right)_{\text {True }}}=15.1$. 
Gumbel distribution parameters for $\left(U_{n}^{\oplus}\right)_{\text {Ret }}$ are made by making use of Equation 8 in Section $2 \mathrm{~b}$. The variance of $\left(\overline{U_{2}}+u_{2}^{\prime}\right)_{\text {Ret }}$ will be affected by the error term, due to variance of $\left(w_{2}^{\prime}-w_{1}^{\prime}\right)$ and the covariance of $\left(\overline{U_{2}}+u_{2}^{\prime}\right)$ with $\left(w_{2}^{\prime}-w_{1}^{\prime}\right)$. The problem of solving for the variance of $\left(\overline{U_{2}}+u_{2}^{\prime}\right)_{\text {Ret }}$ and for the Gumbel parameters of $\left(U_{n}^{\oplus}\right)_{\text {Ret }}$ can be simplified by assuming that the covariance between $\left(\overline{U_{2}}+u_{2}^{\prime}\right)$ and $\left(w_{2}^{\prime}-w_{1}^{\prime}\right)$ is zero. That is, the difference in vertical velocities over the $b_{1}$ - and $b_{2}$-beams is assumed to be uncorrelated with perturbations in the horizontal velocity. This assumption will be made, causing the variance of $\left(\overline{U_{2}}+u_{2}^{\prime}\right)_{\text {Ret }}$ to be greater than that of $\left(\overline{U_{2}}+u_{2}^{\prime}\right)_{T r u e}$. It is the increased variance of $\left(\overline{U_{2}}+u_{2}^{\prime}\right)_{\text {Ret }}$ that results in a positive bias in $\overline{\left(U_{n}^{\oplus}\right)_{\text {Ret }}}$.

The additional variance in $\left(\overline{U_{2}}+u_{2}^{\prime}\right)_{\text {Ret }}$ will be dependent on the variance of the difference in vertical velocities over the two beams, $\left(w_{2}^{\prime}-w_{1}^{\prime}\right)$. The variance of this difference will be referred to as the variance of the residual, Var[Resid] because it could be zero if the vertical velocities over the two beams were identical. In the case where $w_{2}^{\prime}$ is known the retrieval equation will not include the error term.

The variance of $\left(\overline{U_{2}}+u_{2}^{\prime}\right)_{\text {Ret }}, \operatorname{Var}\left[\mathbf{U}_{\text {Ret }}\right]$, is then obtained by summing the variance of $\left(\overline{U_{2}}+u_{2}^{i}\right)_{T \text { True }}$, or $\sigma_{\text {True }}^{2}$, with the product of $\operatorname{Var}[$ Resid] and the square of the amplifying cotangent term:

$$
\operatorname{Var}\left[\mathbf{U}_{\mathrm{Ret}}\right]=\sigma_{\text {True }}^{2}+\operatorname{Var}[\operatorname{Resid}] \cdot \operatorname{Cot}^{2}(\Theta)
$$

For the case where $\operatorname{Var}\left[\right.$ Resid] is $(1.0)^{2}, \Theta=15^{\circ}$, and the variance of $\left(\overline{U_{2}}+u_{2}^{\prime}\right)_{\text {True }}$ is $(2.0)^{2}, \operatorname{Var}\left[\mathbf{U}_{\text {Ret }}\right]$, becomes 17.9 , a substantial increase. 
The average value for the $\left(U_{n}^{\oplus}\right)_{\text {Ret }}$ can now be calculated from

$$
\overline{\left(U_{n}^{\oplus}\right)_{\text {Ret }}}=\overline{U_{2}}+\sigma_{\text {Ret }} * \mathbf{G}_{\mathbf{c}} .
$$

where $\sigma_{\text {Ret }}=\left\{\operatorname{Var}\left[\mathbf{U}_{\text {Ret }}\right]\right\}^{1 / 2}$. From Equation 18 we find $\overline{\left(U_{n}^{\oplus}\right)_{\text {Ret }}}=20.8$, using $\overline{U_{2}}=10$, $\sigma^{2}=4.0$, and $\mathrm{n}=100$, as above. $\overline{\left(U_{n}^{\oplus}\right)_{\text {Ret }}}$ is $\sim 38 \%$ higher than $\overline{\left(U_{n}^{\oplus}\right)_{\text {True }}}$, which was found to be 15.1 .

Figure 2 shows modeled probability density functions (pdfs) of $\left(\overline{U_{2}}+u_{2}^{\prime}\right)_{\text {True }}$, $\left(U_{n}^{\oplus}\right)_{\text {True }}$, and $\left(U_{n}^{\oplus}\right)_{\text {Ret }}$ for the case outlined above. The $\left(U_{n}^{\oplus}\right)_{\text {rrue }}$ and $\left(U_{n}^{\oplus}\right)_{\text {Ret }}$ distributions are shifted significantly to the right of the underlying distribution of $\left(\overline{U_{2}}+u_{2}^{\prime}\right)_{\text {True }}$, because the theoretical peak value distributions were determined for sample sizes of 100 . It is also evident that the distribution of $\left(U_{n}^{\oplus}\right)_{\text {Ret }}$ is somewhat wider than that of $\left(U_{n}^{\oplus}\right)_{\text {True }}$. For the case where $\Theta=30^{\circ}, \operatorname{Var}\left[\mathbf{U}_{\text {Ret }}\right]$ would be reduced to 7.0 and the bias would be reduced to $11 \%$. The bias decreases to zero as $\Theta$ approaches $90^{\circ}$, as expected.

The influence of the variance of $\left(w_{2}^{\prime}-w_{1}^{\prime}\right)$ in enhancing $\overline{\left(U_{n}^{\oplus}\right)_{\text {Ret }}}$ above $\overline{\left(U_{n}^{\oplus}\right)_{\text {True }}}$ can be visualized by graphing the percent bias of $\overline{\left(U_{n}^{\oplus}\right)_{\text {Ret }}}$ in a two-dimensional, normalized coordinate system of $\sigma_{\text {True }} / \overline{U_{2}}$ versus $\sigma_{\text {Resid }} / \overline{U_{2}}$. Figure 3 shows contours of the percent-bias,

$$
\% \text { Bias }=100 *\left(\overline{\left(U_{n}^{\oplus}\right)_{\text {Ret }}}-\overline{\left(U_{n}^{\oplus}\right)_{\text {True }}}\right) /\left(\overline{\left.U_{n}^{\oplus}\right)_{\text {True }}}\right.
$$

for the case where $n=100$, and theta $=15$ degrees. The greatest bias is found when the residual variance in vertical velocity is large compared to the variance in horizontal velocity. The bias becomes small when the residual variance in vertical velocity is small. 
Figure 4 shows contours of the percent bias for the case where $n=100$ and $\Theta=30^{\circ}$. For fixed values of $\sigma_{\text {Resid }} / \overline{U_{2}}$ and $\sigma_{\mathrm{T}} / \overline{U_{2}}$, the percent bias with $\Theta=30^{\circ}$ is less than half that for $\Theta=15^{\circ}$ (Fig. 3) because of the strong $\Theta$ dependence of the $\cot ^{2}(\Theta)$ factor.

\section{Summary and Conclusions}

Numerous previous studies have shown that Doppler profilers are capable of providing accurate retrievals of average horizontal velocities in the atmosphere and underwater. However, estimates of peak horizontal velocities from collections of instantaneous retrievals are susceptible to a positive bias, due to turbulent vertical motions in the medium of interest.

An idealized Doppler profiler configuration was combined with a statistical model of turbulent motions and a simple retrieval algorithm to illustrate the nature of the bias and its magnitude. The results revealed that unresolved vertical motions contaminate the instantaneous retrievals because of limitations inherent in the beam configuration of typical profilers. The retrieved instantaneous horizontal velocities are more variable than the true instantaneous velocities, resulting in a positive bias when the peak retrieved value is chosen.

The bias in peak horizontal velocity can be characterized in terms of turbulent properties of the flow. Errors in the measurement of the vertical velocity would also contribute to the bias. These findings suggest that an average correction could be applied to the retrieved peak values. However, the correction would be statistical in nature and would not necessarily improve the precision of individual retrieved peak values. 


\section{APPENDIX A}

\section{List of Symbols}

\begin{tabular}{|c|c|}
\hline$a_{n}$ & Gumbel normalization constant \\
\hline$b_{n}$ & Gumbel normalization constant \\
\hline$b_{1}$-beam & Vertical beam \\
\hline $\mathrm{b}_{2}$-beam & Oblique beam \\
\hline $\mathrm{D}_{1}$ & Distance along $b_{1}$-beam axis \\
\hline $\mathrm{D}_{2}$ & Distance along $b_{2}$-beam axis \\
\hline $\mathrm{Eu}$ & Euler's constant \\
\hline $\mathrm{G}_{\mathrm{C}}$ & Gumbel constant $\left(a_{n}+b_{n} \cdot E u\right)$ \\
\hline $\mathrm{G}(\mathrm{x})$ & Gumbel probability density function \\
\hline $\mathrm{H}$ & Height above the profiler \\
\hline $\mathrm{n}$ & Sample size \\
\hline $\mathrm{p}$ & Location of profiler \\
\hline Resid & Residual quantity \\
\hline$R$ & Radial velocity \\
\hline$r^{\prime}$ & Perturbation $R$ \\
\hline$U$ & Horizontal velocity \\
\hline$u^{\prime}$ & Perturbation horizontal velocity \\
\hline $\operatorname{Var}[\mathrm{x}]$ & Variance of the variable $\mathrm{x}$ \\
\hline $\mathrm{V}_{1}$ & Radial velocity along $b_{1}$-beam axis \\
\hline$V_{2}$ & Radial velocity along $b_{2}$-beam axis \\
\hline$W$ & Vertical velocity \\
\hline
\end{tabular}


$w$

$\Theta$

$\lambda$

$\xi$

$\sigma$

$-$

$\oplus$

1

2

Ret

True
Perturbation vertical velocity

Angle between vertical and oblique beams

Scale parameter for Gumbel distribution

Location parameter for Gumbel distribution

Standard deviation

Overbar indicates ensemble average value

Prime indicates perturbation value

Indicates a.peak value

Subscript 1 indicates vertical beam

Subscript 2 indicates oblique beam

Subscript Ret indicates a retrieved value

Subscript True indicates a true value 
Notice

Mention of a copyrighted, trademarked or proprietary product, service, or document does not constitute endorsement thereof by the author, ENSCO, Inc., the AMU, the National Aeronautics and Space Administration, or the United States Government. Any such mention is solely to inform the reader of the resources used to conduct the work reported herein. 
Acknowledgements

The authors thank Ms. Winifred Lambert and Dr. Greg Taylor for their useful comments on this project. 


\section{REFERENCES}

Abramowitz, M., and I. A. Stegun, 1965: Handbook of Mathematical Functions. Dover Publications, Inc., New York, 1046 pp.

Chereskin, T. K., D. Halpern, and L. A. Regier, 1987: Comparison of shipboard acoustic Doppler current profiler and moored current measurements in the equatorial Pacific. J. Atmos. Oceanic Tech., 4, 742-747.

Coles, S., 2001: An Introduction to Statistical Modeling of Extreme Values, Springer Series in Statistics, $208 \mathrm{pp}$.

Crescenti, G. H., 1997: A look back at two decades of Doppler SODAR comparison studies. Bull. Amer. Meteor. Soc., 78, 651-673.

Gilboy, T.P., T.D. Dickey, D.E. Sigurdson, X. Yu, and D. Manov, 2000. An intercomparison of current measurements using a VMCM, and $\mathrm{ADCP}$, and a recently developed acoustic current meter. J. Atmos. Oceanic Tech., 17, 561-574.

Kramar, V. F., and R. D. Kouznetsov, 2002: A new concept for estimation of turbulent parameter profiles in the ABL using sodar data. J. Atmos. Oceanic Tech., 19, 12161224.

Lu, Y., and R. G. Lueck, 1999: Using a broadband ADCP in a tidal channel: Part II: Turbulence. J. Atmos. Oceanic Tech., 16, 1568-1579.

May, P.T., T. Sato, M. Yamamoto, S. Kato, T. Tsuda, and S. Fukao, 1989: Errors in the determination of wind speed by Doppler radar. J. Atmos. Oceanic Tech., 6, 235-242. 
Mitsuta, Y., and O. Tsukamoto, 1989: Studies on spatial structure of wind gust. J. Appl. Meteor., 28,1155-1160.

Peterson, V. L., 1988: Wind Profiling: The History, Principles, and Ap̄plicatiōñ of Clear-Air Doppler Radar. Tycho Technology, Inc., Boulder, CO, 66 pp.

Van Zandt, T. E., 2000: A brief history of the development of wind-profiling or MST radars. Ann. Geophysicae, 18, 740-749.

Woodward, W.E. and Appell, G.F., 1986: Current velocity measurements using acoustic Doppler backscatter: A review. IEEE J. Oceanic Eng., OE-11, 3-6. 


\section{Figure Captions}

Figure 1. Schematic of an idealized 2-axis Doppler profiler system

Figure 2. Modeled pdfs of horizontal velocities illustrating Gumbel distributions of true and retrieved peaks derived from a background distribution that is Gaussian.

Distribution parameters have been chosen to give an average background velocity of 10 , an average value of 15.1 for the true peaks and a bias of $38 \%$ for the retrieved peaks. The standard deviation of true horizontal velocities is 2.0 and the standard deviation of the residual vertical velocity is 1.0 .

Fig. 3. The percent bias in $\overline{\left(U_{n}^{\oplus}\right)_{\text {Ret }}}$ as a function of $\sigma_{\text {True }} / \bar{U}$ and $\sigma_{\text {Resid }} / \bar{U}$, for $\mathrm{n}=100$ and $\Theta=15^{\circ}$, based on the analytical models for horizontal and vertical velocity presented in Section 3.

Fig. 4. As in Fig.3, but for $\Theta=30^{\circ}$. 


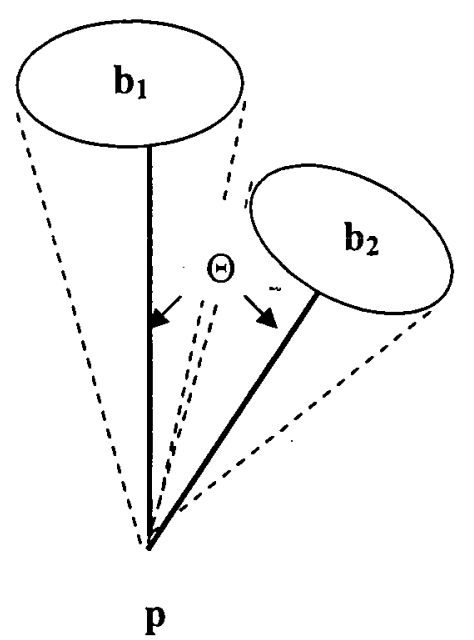

Figure 1. Schematic of an idealized 2-axis Doppler profiler system. 


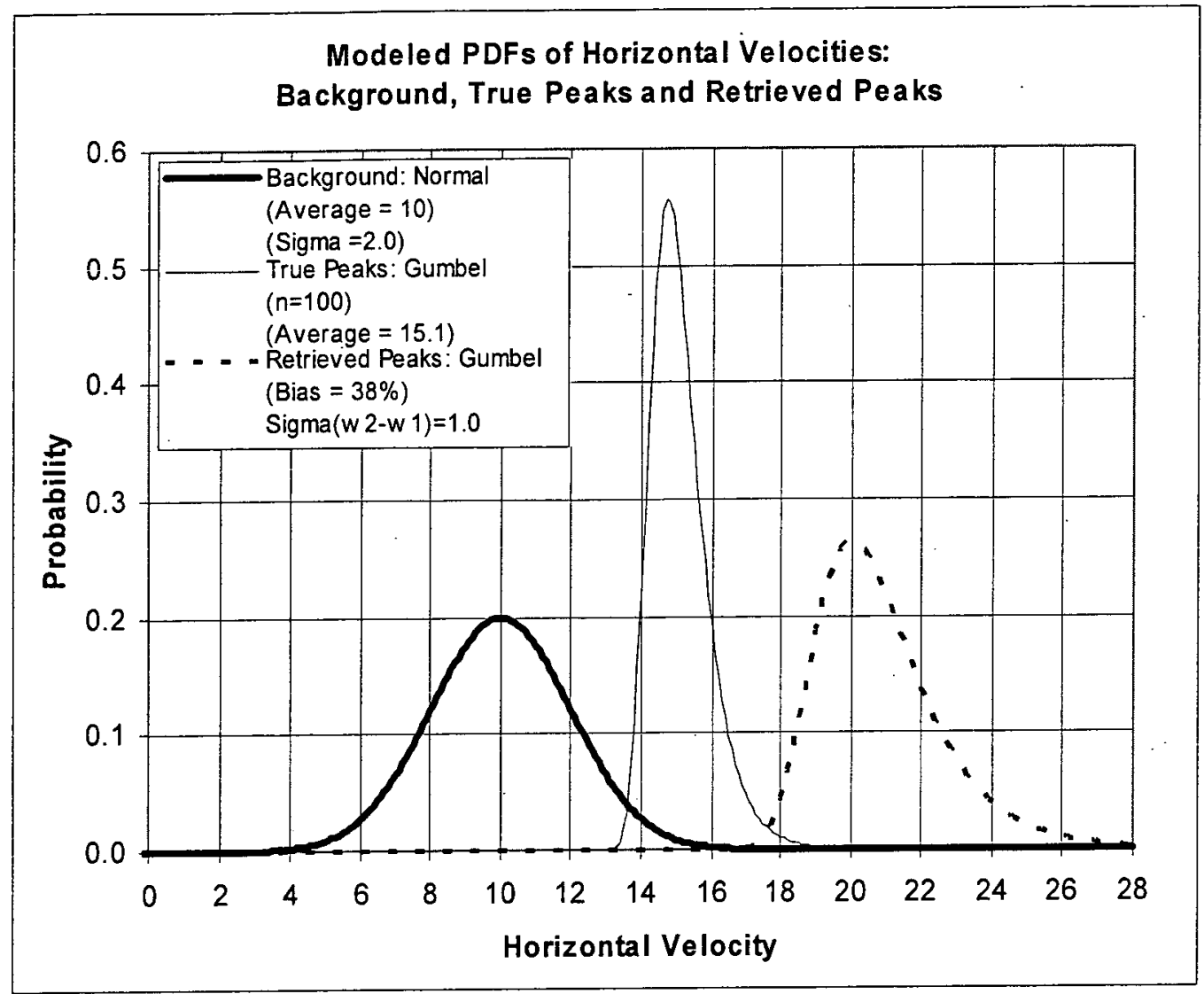

Figure 2. Modeled pdfs of horizontal velocities illustrating Gumbel distributions of true and retrieved peaks derived from a background distribution that is Gaussian. Distribution parameters have been chosen to give an average background velocity of 10 , an average value of 15.1 for the true peaks and a bias of $38 \%$ for the retrieved peaks. The standard deviation of true horizontal velocities is 2.0 and the standard deviation of the residual vertical velocity is 1.0 . 
Bias: Average Retrieved $\left(U^{*}\right): n=100$, theta $=15$

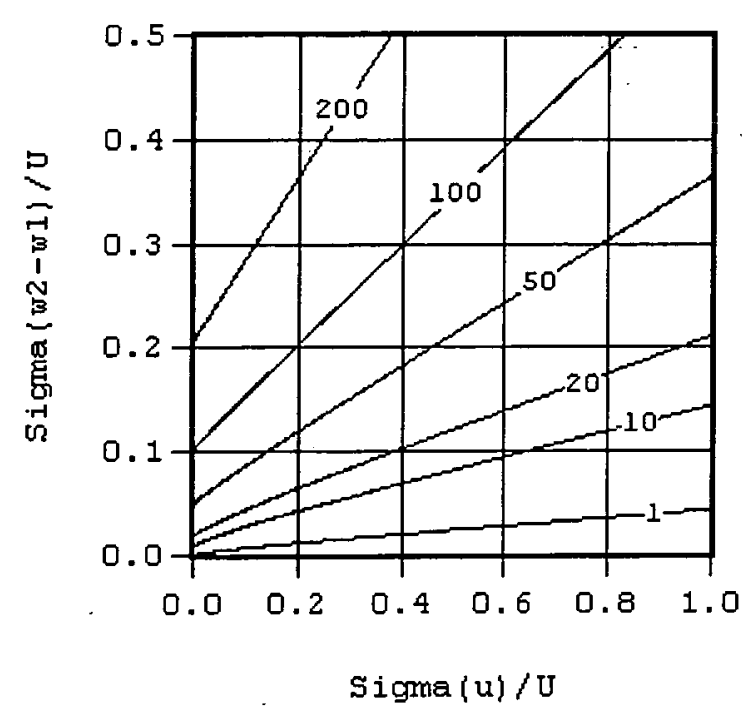

Figure 3. The percent bias in $\overline{\left(U_{n}^{\oplus}\right)_{\text {Ret }}}$ as a function of $\sigma_{\text {True }} / \bar{U}$ and $\sigma_{\text {Resid }} / \bar{U}$, for $\mathrm{n}=100$ and $\Theta=15^{\circ}$, based on the analytical models for horizontal and vertical velocity presented in Section 3. 
* Bias: dverage Retrieved (U*): $n=100$, theta=30

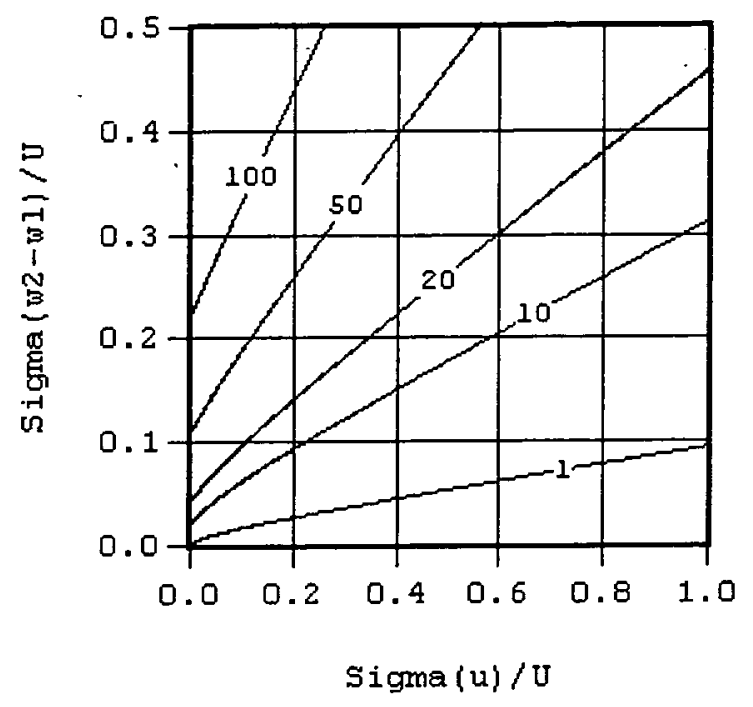

Fig. 4. As in Fig.3, but for $\Theta=30^{\circ}$. 